\title{
Towards Reducing Carbon Content in Silicon/Carbon Anodes for Lithium Ion Batteries
}

\author{
Yuzi Zhang ${ }^{1}$, Yue Pan ${ }^{2}$, Yanjing Chen ${ }^{1}$, Brett L. Lucht ${ }^{2}$, Arijit Bose ${ }^{1, *}$ \\ ${ }^{1}$ Department of Chemical Engineering, ${ }^{2}$ Department of Chemistry, University of Rhode \\ Island, Kingston, Rhode Island, 02881
}

* Corresponding author: Arijit Bose, bosea@uri.edu, 401-874-2804

Key words: Reduced carbon; Silicon anodes; Lithium ion batteries 


\begin{abstract}
Conducting carbon is added to electrodes of lithium ion batteries (LIBs) to provide electrical conductivity. Because this carbon does not contribute to capacity, there is a drive towards decreasing its content with a goal of lowering the mass of the electrode. Reduced graphene oxide (RGO) has a high electrical conductivity, and is a potential alternative to traditionally used conductive carbon black (CB) in anodes for LIBs. Because of its high aspect ratio, RGO is expected to form a conducting network at lower volume loadings than CB. We report the use of this concept to significantly reduce carbon loading in silicon-carbon anodes for LIBs formed by emulsion-templating. Anodes with $1 \mathrm{wt} \% \mathrm{RGO}$ and $14 \mathrm{wt} \% \mathrm{CB}$ (15 wt\% total carbon) showed specific capacities and capacity retentions that were comparable to anodes with $30 \mathrm{wt} \% \mathrm{CB}$ with or without RGO. The capacity retention was significantly lower for anodes with 15 wt\% total carbon that had no RGO. Cryo-SEM and SEM images, and electrochemical impedance spectroscopy, confirmed the formation of a conducting carbon network at 15 wt\% total carbon loading when 1 wt $\%$ of the CB was replaced with RGO, and the lack of a well-connected network without the RGO.
\end{abstract}




\section{Introduction}

Lithium ion batteries (LIBs) with high energy density are desirable for use in advanced electronic devices and electric vehicles. ${ }^{1-6}$ Because high electron conductivity is required for the anode and cathode, conductive carbon black (CB) is usually added to either electrode. The conductive carbon additive does not contribute to electrode capacity, and one important goal in LIBs electrode design is to minimize its use, thus increasing the specific mass capacity of the battery. ${ }^{7-10}$

The volume fraction loading at the percolation threshold indicates the minimum amount of conducting filler needed to impart conductivity to an insulating matrix. ${ }^{11-12}$ For monodispersed solid spheres, the percolation threshold $\emptyset_{\text {sphere }}=0.29 .{ }^{13}$ When fractal particles, such as CB are deployed, their effective volume fraction in a suspension can be estimated using the following equation, ${ }^{14-15}$

$$
\frac{\emptyset_{\text {eff }}}{\emptyset_{\mathrm{o}}}=\left(D / D_{0}\right)^{3-f},
$$

where $\emptyset_{\text {eff }}$ and $\emptyset_{0}$ are the effective and actual volume fractions of the CB particles in the suspension, $D$ is the hydrodynamic diameter of the fractal CB particle $(\sim 120 \mathrm{~nm}), D_{0}$ is diameter of the primary particle in the CB ( 20 nm) and $f$ is the mass fractal dimension of the particle. $D / D_{0}=6$, and the fractal dimension $f$ for the CB particles is $2.2 .{ }^{16} \mathrm{Eq}$. (1) shows that the effective volume fraction occupied by these CB particles would be $\sim 4$ times the volume fraction occupied by the same mass of spherical particles. This lower material requirement is one of the key reasons behind the use of fractal $C B$ rather than dense spherical carbon particles to enhance electrode conductivity. For sheet-like materials, the percolation threshold $\emptyset_{\text {sheet }} \sim \emptyset_{\text {sphere }} / A R{ }^{13,17}$ The aspect ratio $A R=L / t$, 
where $L$ is the lateral dimension, and $t$ is the sheet thickness. $A R$ for reduced graphene oxide (RGO) is typically of the order of $10^{3}-10^{4}$. Given the much lower percolation threshold for sheet-like materials of high aspect ratio and the high electrical conductivity of RGO, we hypothesized that replacing some of the CB with RGO would reduce the total carbon used in an electrode without sacrificing electrical conductivity. We examined the hypothesis in this work.

In previous work, we used emulsion-templating for forming silicon-based anodes that showed good electrochemical performance ${ }^{18} \mathrm{CB}$ particles were used to stabilize emulsions as well as form a conducting carbon network. The organization of silicon nanoparticles (SiNPs) into porous carbon cages formed by the CB allowed expansion and contraction of the SiNPs during lithiation and delithiation without pulverization and without disrupting the conducting carbon network. In this paper we show that when a small fraction of the conducting carbon comes from RGO, the total carbon content of an anode prepared by emulsion-templating can be lowered significantly without sacrificing electrochemical performance.

\section{Experiments}

\section{Preparation of anodes}

SiNPs (average diameter $\sim 50 \mathrm{~nm}$ ) were purchased from Alfa Aesar. A para-amino benzoic acid-terminated CB (specific surface area of CB $\sim 200 \mathrm{~m}^{2} / \mathrm{g}$ ) suspension in water at $\mathrm{pH} 7.5$ was obtained from Cabot Corporation. While the surface treatment for this $\mathrm{CB}$ introduces electrical resistance, the surface functionality is important for stabilizing emulsions. At neutral $\mathrm{pH}$, the carboxyl groups on the $\mathrm{CB}$ particles are 
deprotonated. These CB particles are highly hydrophilic and form a stable suspension in water. Reduced graphene oxide particles (RGO) were obtained from Graphene Supermarket. The RGO particles have a specific surface area of $\sim 833 \mathrm{~m}^{2} / \mathrm{g}$ and a carbon/oxygen ratio of 10.5 . The average lateral dimension of the RGO particles is 4 $\mu \mathrm{m}$.

The SiNP/CB/RGO anodes were prepared by an emulsion-templating method shown in Figure 1. $1 \mathrm{M} \mathrm{HCl}$ was added to a mixed $\mathrm{CB}$ and $\mathrm{RGO}$ suspension, or only a $\mathrm{CB}$ containing suspension, until the $\mathrm{pH}$ reached 3.3. The addition of the acid protonated some of the surface carboxylate groups on the $\mathrm{CB}$, and made the particles partially hydrophobic. These CB particles favor oil-water interfaces, and stabilize oil-in-water emulsions. ${ }^{19}$ In addition, the partially hydrophobic CB particles formed a network in the aqueous phase. ${ }^{19}$ When RGO was also present in the suspension, the network contained both entities.

A 2.5 wt\% suspension of SiNPs in octane was sonicated for 1 hour, and then mixed with the acid-mediated CB and RGO suspension by vortexing for 3 min at $3000 \mathrm{rpm}$. The volume ratio of the SiNPs/octane suspension to the $\mathrm{CB} / \mathrm{RGO} /$ water suspension was 2:5. An 'octane-in-water' emulsion was formed after vortexing. A $20 \mathrm{mg} / \mathrm{ml}$ aqueous binder solution containing polyacrylic acid (PAA) and carboxymethyl cellulose $(\mathrm{CMC})$ in a 1:1 mass ratio was added to the emulsion and vortexed for 5 min. ${ }^{20-21}$ The volume of the PAA/CMC solution was adjusted so that the final concentration of binder was $10 \%$ of the dried anode mass consisting of SiNP, CB, RGO and binder. The 
emulsion was transferred on to a CR2032 stainless steel coin cell and dried overnight at $50^{\circ} \mathrm{C}$ under a vacuum, thus removing all the octane and water. The areal loading on the coin cell was $\sim 0.54 \mathrm{mg} / \mathrm{cm}^{2}$ and the final dried thickness was $\sim 100-150 \mu \mathrm{m}$. We examined samples at $30 \mathrm{wt} \%$ and $15 \mathrm{wt} \%$ total carbon, measured for the dry state. Two classes of samples were prepared at each total carbon loading, one with $1 \mathrm{wt} \% \mathrm{RGO}$ and the other without RGO. Because the binder concentration was fixed at $10 \mathrm{wt} \%$, reducing the total carbon content meant that the SiNP wt\% was increased.

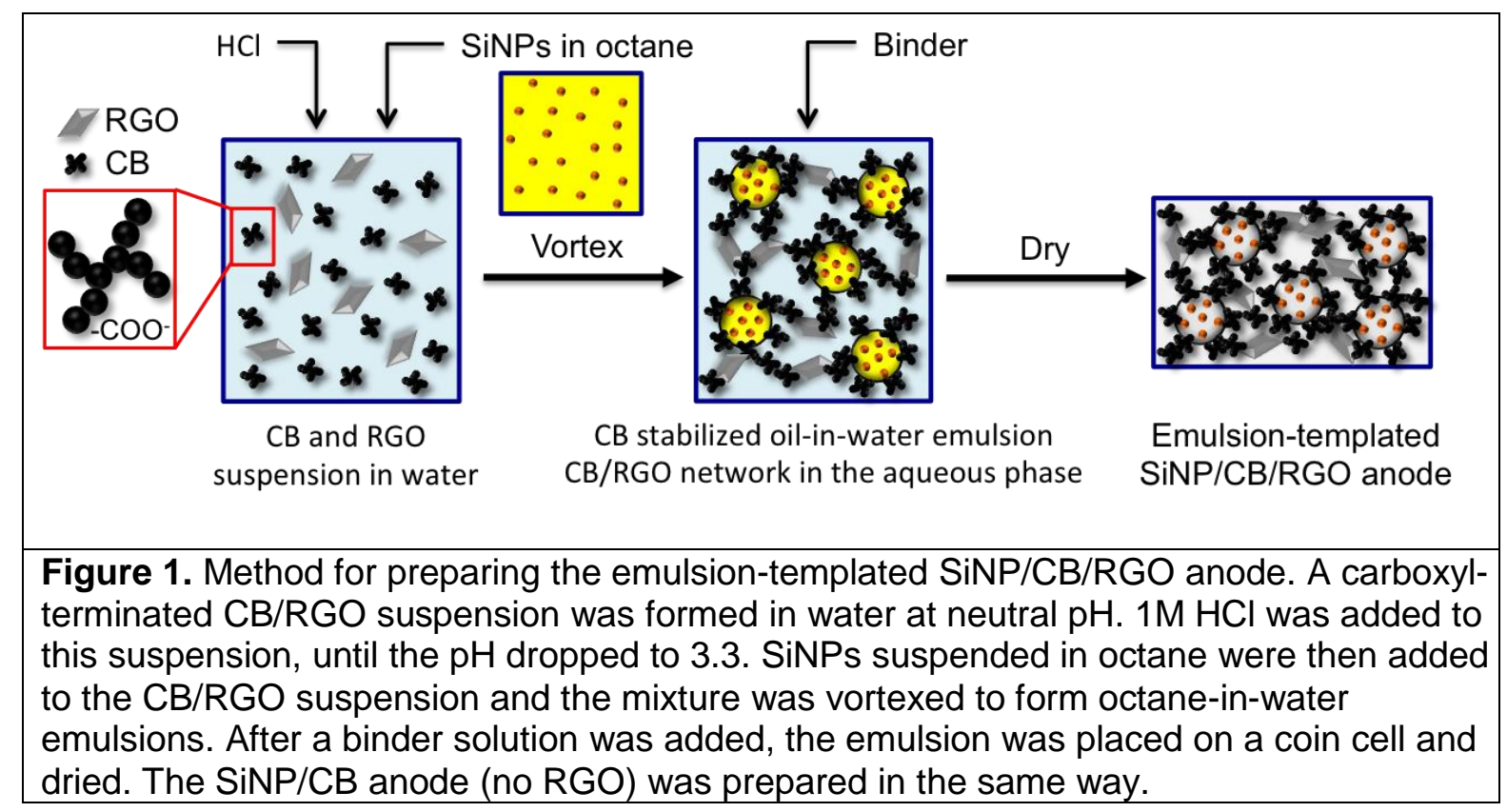

\section{Electrochemical characterization}

CR2032 coin cells were assembled in an argon-filled glovebox that had a water vapor concentration of less than $1 \mathrm{ppm}$. The SiNP-based anodes served as the working electrodes. Lithium foil was employed as the counter electrode. The electrolyte, supplied by BASF, was a mixture of $1.0 \mathrm{M} \mathrm{LiPF}_{6}$ in ethylene carbonate $(\mathrm{EC})$ / diethyl 
carbonate (DEC) / fluoroethylene carbonate (FEC) at a volumetric ratio of 45:45:10. A polypropylene microporous membrane, Celgard 2325, along with a glass fiber membrane, was used as the separator. Galvanostatic charge/discharge tests were performed on a BST8-WA battery cycler $(5 \mathrm{~V} / 1 \mathrm{~mA})$ over the potential range $0.005-1.5$ V vs. $\mathrm{Li}^{+} / \mathrm{Li}$, at room temperature, with a cycle rate of $0.1 \mathrm{C}$ for 3 cycles followed by $0.2 \mathrm{C}$ for 47 cycles, for a total of 50 cycles. The current density, CD, required for each charge or discharge rate was calculated using the following equation:

$\mathrm{CD}=($ Areal loading) (wt\% SiNP) (Si theoretical capacity)/(cycle time)

As an example, for a $60 \mathrm{wt} \%$ SiNP sample, Eq.(2) implies a current density of 69 $\mu \mathrm{A} / \mathrm{cm}^{2}$ for charge/discharge rates of $0.1 \mathrm{C}$.

Electrochemical Impedance Spectroscopy (EIS) was carried out on a Princeton Instruments V3 Potentiostat, at room temperature. The impedance of each half cell was measured at the $3^{\text {rd }}$ cycle, both in the lithiated state, where the cell potential was $0.15 \mathrm{~V}$, and in the delithiated state where the cell potential was 1.13V. In addition, EIS spectra were obtained after 50 cycles for the delithiated state, where the cell potential was 1.15 V. The amplitude of the sinusoidal voltage perturbation for EIS was $10 \mathrm{mV}$ and the frequency range was $20 \mathrm{mHz}-100 \mathrm{KHz}$. 


\section{Cryogenic Scanning Electron Microscopy (Cryo-SEM)}

The microstructures present in the SiNP/CB/RGO and SiNP/CB emulsions were imaged by cryo-SEM using a Gatan Alto 2500 cryo preparation system attached to a Zeiss Sigma VP field emission scanning electron microscope. A small drop containing the emulsion was placed on the sample holder. The sample was frozen rapidly by plunging into liquid nitrogen, then transferred into the preparation chamber maintained at $-130^{\circ} \mathrm{C}$. A flat-edge cold knife in the chamber was utilized to fracture the frozen sample. The chamber was then warmed to $-100^{\circ} \mathrm{C}$ for 2 minutes to enhance the surface topology of the sample by differential sublimation. After cooling the chamber back to $-130^{\circ} \mathrm{C}$, the sample was sputter coated with platinum for $60 \mathrm{~s}$, then transferred onto a liquid nitrogen cooled cold stage for imaging at $2 \mathrm{kV}$.

\section{Scanning Electron Microscopy (SEM)}

Secondary electron imaging on the Zeiss Sigma VP field emission scanning electron microscope was used at $10 \mathrm{kV}$ to visualize the dried emulsion samples.

\section{Results and discussion}

We confirmed that the emulsions we prepared were all of the oil-in-water type by adding drops of water or octane to the emulsion. ${ }^{22}$ The water drop blended in readily, but the octane drop did not. 
The electrochemical performance of the SiNP/CB/RGO and SiNP/CB containing anodes are shown in Figure 2. They represent an average from 3 different cells, and the spread in the data are shown as error bars. The specific capacities at the delithiated states of SiNP/CB/RGO and SiNP/CB anodes for $30 \mathrm{wt} \%$ and $15 \mathrm{wt} \%$ total carbon are shown in Figure 2A. All specific capacity and wt\% values were calculated based on the total mass of SiNP, CB, RGO and binder. At $30 \mathrm{wt} \%$ total carbon, after 50 cycles, the delithiation capacities were $1287 \mathrm{mAh} / \mathrm{g}$ and $1244 \mathrm{mAh} / \mathrm{g}$ for the $\mathrm{SiNP/CB/RGO}$ and SiNP/CB anodes respectively. At this carbon loading there was little difference between the performance of anodes with and without RGO. However for 15 wt\% total carbon, the performance of the anode with RGO was significantly better than the one without - the delithiation capacities at 50 cycles were $1370 \mathrm{mAh} / \mathrm{g}$ for the SiNP/CB/RGO and $1044 \mathrm{mAh} / \mathrm{g}$ for the SiNP/CB anode.

We allowed for initial SEI formation by using charge/discharge rates of $0.1 \mathrm{C}$ for the first 3 cycles. The capacity retentions of all the anodes from the $4^{\text {th }}$ cycle onwards, where we used charge/discharge rates of $0.2 \mathrm{C}$, normalized by their respective capacities at the end of the $4^{\text {th }}$ cycle, are shown in Figure $2 \mathrm{~B}$. The anodes with $30 \mathrm{wt} \%$ carbon with and without RGO had capacity retentions of $83 \%$ and $80 \%$ respectively. At this total carbon loading, the replacement of some of the CB with RGO did not provide any advantage. At 15 wt $\%$ total carbon with 1 wt $\%$ RGO, the capacity retention after 50 cycles was $77 \%$, comparable to the retention for the $30 \mathrm{wt} \%$ carbon loading. However, with no RGO, the $15 \mathrm{wt} \%$ carbon anode showed a capacity retention of only $61 \%$ after 50 cycles. Thus, the total carbon loading can be reduced by a factor of 2, from $30 \mathrm{wt} \%$ 
to $15 \mathrm{wt} \%$, without paying a capacity stability penalty, if only a small amount of RGO is used in combination with CB.
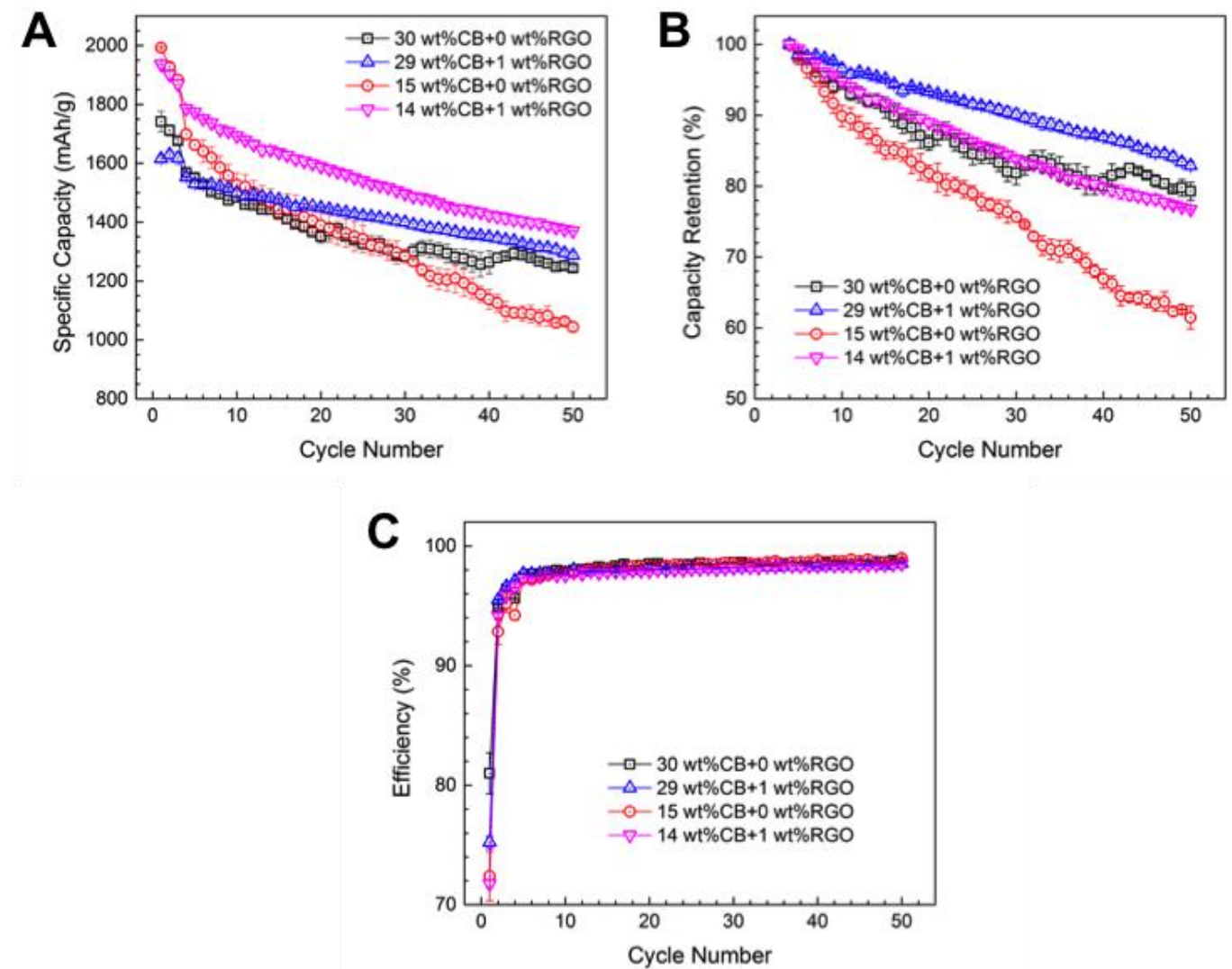

Figure 2. Electrochemical characterization of the emulsion-templated SiNP-based anodes. (A) Delithiation capacity, (B) Delithiation capacity retention (normalized by their respective capacities at the $4^{\text {th }}$ cycle), (C) Coulombic efficiency of SiNP/CB/RGO and $\mathrm{SiNP} / \mathrm{CB}$ anodes at $30 \mathrm{wt} \%$ and $15 \mathrm{wt} \%$ total carbon, at a charge/discharge rate of $0.1 \mathrm{C}$ for the first 3 cycles followed by $0.2 \mathrm{C}$ for 47 cycles. The data represent average values from 3 runs. The error bars indicate the maximum spread in the data.

Figure $2 \mathrm{C}$ shows the Coulombic efficiency for all the anodes. The low efficiency for the first 3 cycles is associated with SEI formation. ${ }^{23}$ In the following cycles, the efficiencies varied between $94.5 \%$ and $98.9 \%$, indicating that changing the carbon content in our 
anodes did not lead to modification of lithium ion transport in the cell during charge/discharge cycles.

To further understand the performance of the anodes, electrochemical impedance spectroscopy measurements on SiNP/CB/RGO and SiNP/CB anodes were conducted at delithiated and lithiated states for the 3rd cycle, as well as the delithiated state after 50 cycles. The data for the $3^{\text {rd }}$ cycle provided a window on the state of the anode right after the initial SEI formation. EIS data for the $50^{\text {th }}$ cycle were associated with irreversible changes that occurred to the anode during cycling that affect electrochemical performance.

Nyquist plots, shown in Figures 3A, 3B and 3C, revealed a few important results. All EIS spectra showed a characteristic semicircle corresponding to the charge transfer process, and a linear Warburg component at low frequencies attributed to the diffusion of lithium ions in the anodes. The solution resistance for all the anodes was around 5 ohms $\mathrm{cm}^{2}$. For 3 cycles, the charge transfer resistance at the lithiated state was lower than that in the delithiated state. After 50 cycles, the charge transfer resistance increased significantly. The charge transfer resistances for the anodes with $30 \mathrm{wt} \%$ carbon with and without RGO were comparable, consistent with the capacity data that showed no benefit to replacing some of the CB with RGO at this carbon loading. However, for $15 \mathrm{wt} \%$ carbon, the charge transfer resistance for the anode with $1 \mathrm{wt} \%$ RGO was significantly lower than the one without RGO, and was comparable to the 
resistance from the anodes with $30 \mathrm{wt} \%$ carbon. The lower charge transfer resistance is indicative of a better electron-conducting pathway for the SiNP/CB/RGO than the SiNP/CB anode at this total carbon content. The Warburg impedance in the low frequency regions deviated from the ideal case, which should have a slope of 1 in the Nyquist plot. We believe this is a consequence of the roughness of our anodes which varied from sample to sample. ${ }^{24}$

Figure 3D shows the voltage profile at the $50^{\text {th }}$ cycle for all the samples. The $15 \mathrm{wt} \%$ carbon anode with $1 \mathrm{wt} \%$ RGO showed similar charge and discharge profiles to the anodes with $30 \mathrm{wt} \%$ carbon. The $15 \mathrm{wt} \%$ carbon anode without RGO showed a voltage gap between the charge and discharge curves in the plateau region that was larger than those for any of the other anodes. The larger voltage gap indicates greater polarization during the charge/discharge process, in agreement with the larger charge transfer resistance measured by EIS. 

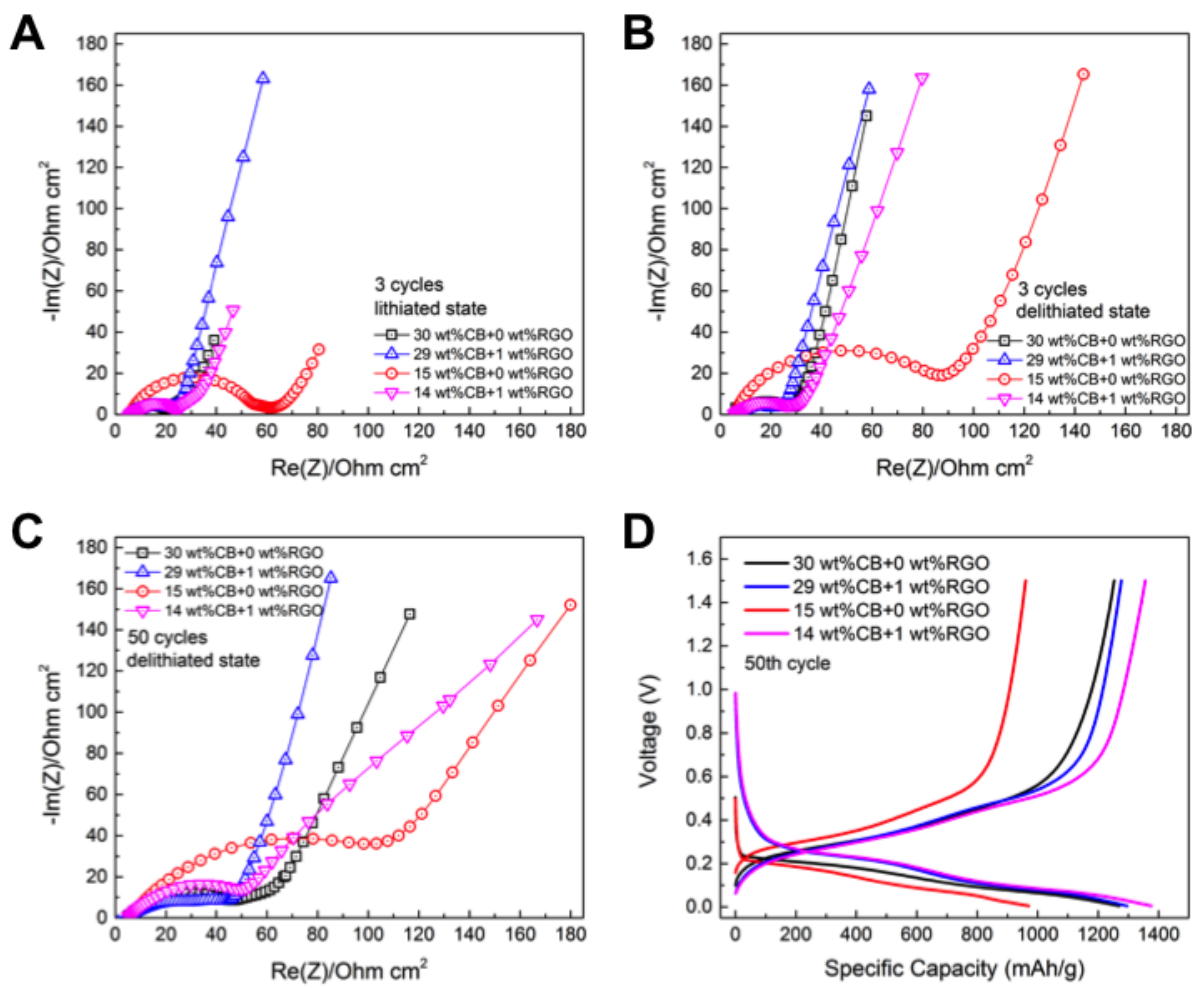

Figure 3. Electrochemical impedance spectra of SiNP/CB/RGO and SiNP/CB anodes (A) At a lithiated state for cycle 3. The open circuit potential was $0.15 \mathrm{~V}$. (B) At a delithiated state for cycle 3. The open circuit potential was 1.13V. (C) At a delithiated state for cycle 50 . The open circuit potential was $1.15 \mathrm{~V}$. (D) Voltage profile at the $50^{\text {th }}$ cycle for all the anodes during lithiation and delithiation. The thickness of the lines correspond to the spread in the data from 3 samples. The voltage gap in the "plateau region' was the largest for the 15 wt\% carbon case with no RGO (red curves).

To further understand the reasons for the variation in electrochemical behavior between the anodes, we sampled the SiNP/CB/RGO and SiNP/CB emulsions using cryo-SEM. Figures 4A and 4B show octane droplets in water in a SiNP/CB/RGO emulsion, stabilized by CB particles, with the SiNPs confined in the oil phase. For the higher carbon content (30 wt\% in dried state), samples with RGO showed a connected carbon network in the aqueous phase (Figures 4C, 4D). The sample without RGO displayed a partially connected network (Figures 4E, 4F). For the emulsion with lower carbon loading (15 wt\% carbon in dried state, with $1 \mathrm{wt} \% \mathrm{RGO}$ ), a network was formed 
in the aqueous phase, consisting of RGO particles and CB (Figures $4 \mathrm{G}, 4 \mathrm{H}$ ). However, for samples with lower carbon loading and no RGO (Figures 4I, 4J), no network was formed. Instead, only some CB aggregates were seen in the aqueous phase.

We also imaged the dried emulsion samples using SEM. Figures $5 \mathrm{~A}, 5 \mathrm{~B}$ and $5 \mathrm{C}$ show a connected network formed in the anodes with $30 \mathrm{wt} \%$ carbon with and without RGO, and 15 wt $\%$ carbon with RGO. The lack of a connected carbon network in the 15 wt $\%$ carbon anode without RGO, shown in Figure $5 \mathrm{D}$, leads to poor electronic conductivity and more extensive capacity fading. This structural information is consistent with the observed capacity behavior and the data from EIS spectra.

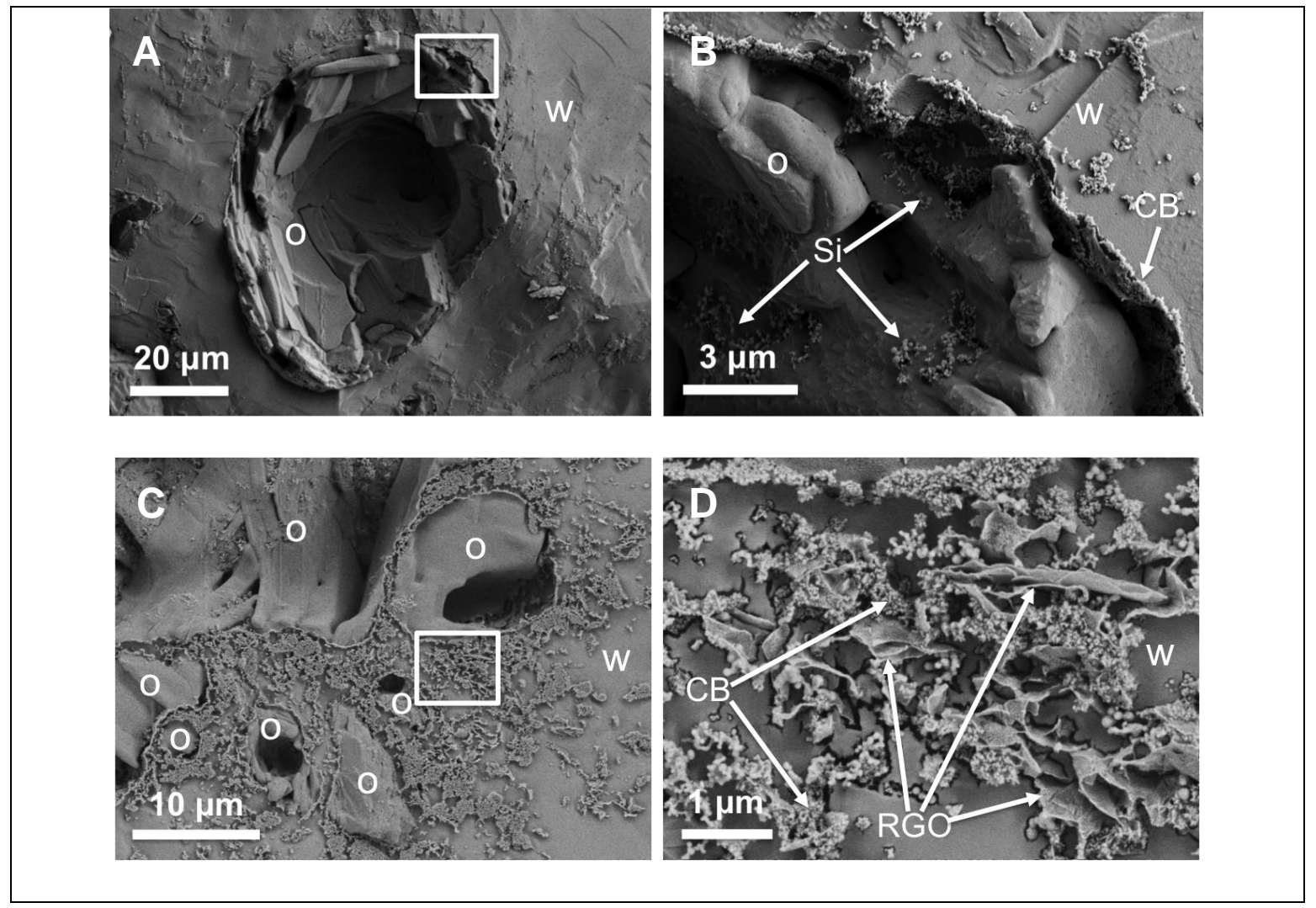




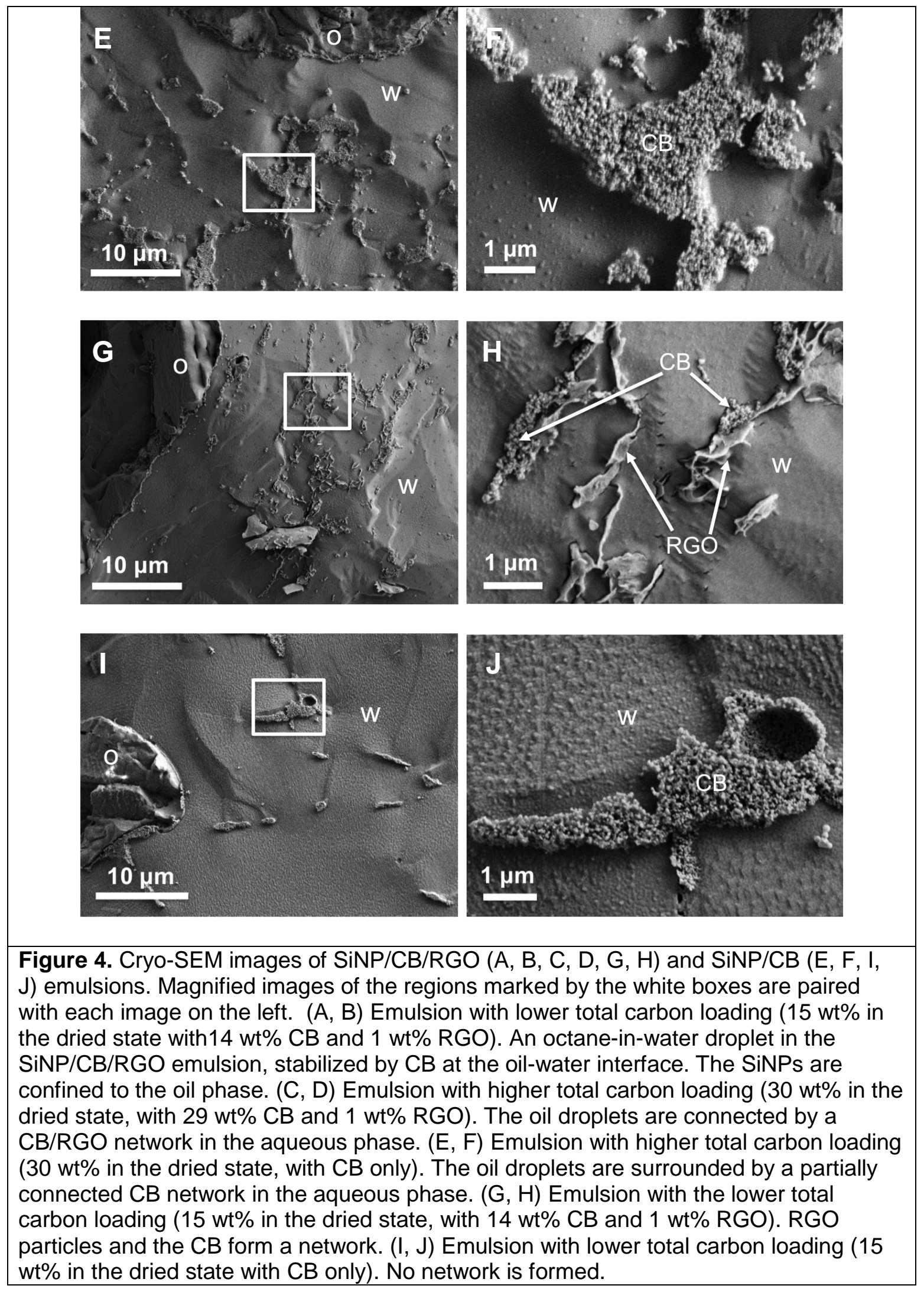



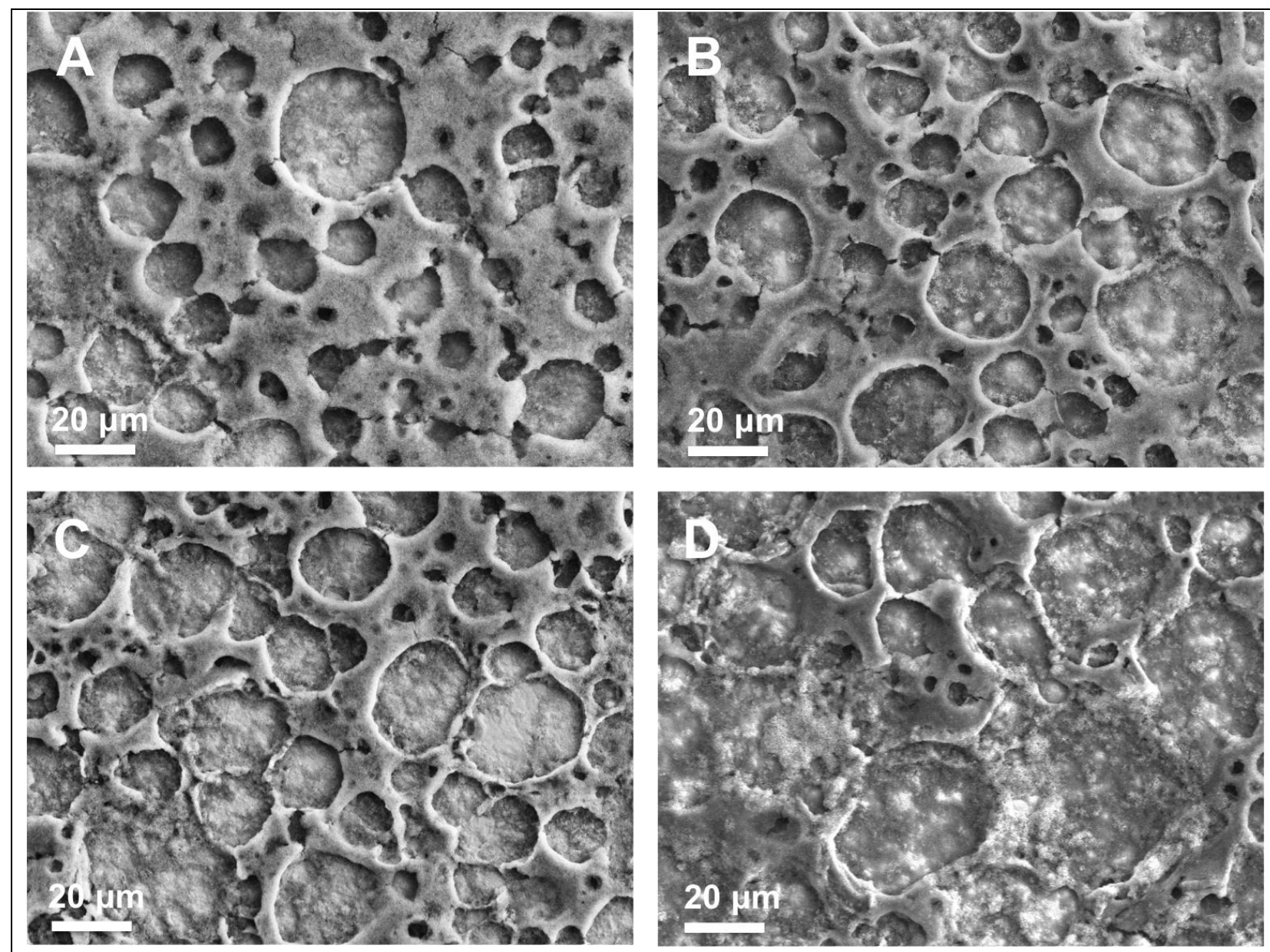

Figure 5. SEM images of the dried emulsions with $30 \mathrm{wt} \%$ total carbon $(A, B)$ and $15 \mathrm{wt} \%$ total carbon (C, D). (A, C) Samples contains 1 wt\% RGO. (B, D) Samples have no RGO. (C) Sample contains 1 wt\% RGO. A connected carbon network is seen in A, B and C. (D) Sample has no RGO. The carbon network is broken in parts, and is only partially connected.

\section{Conclusions}

We demonstrate an effective and simple way to reduce the total carbon content from

$30 \mathrm{wt} \%$ to $15 \mathrm{wt} \%$ in SiNP-based anodes prepared by emulsion-templating. We

introduced 1 wt\% RGO to the electron-conducting phase along with conductive CB

during anode fabrication. For $30 \mathrm{wt} \%$ total carbon loading, the replacement of some of

the CB with RGO did not impact the electrochemical performance of the anode. The 
anode with $15 \mathrm{wt} \%$ total carbon but with $1 \mathrm{wt} \%$ RGO showed electrochemical performance that was comparable to an anode with twice that total carbon loading. Its capacity retention after 50 cycles was much higher than the anode without RGO. The performance difference for the anodes with $15 \mathrm{wt} \%$ total carbon is a consequence of the formation of a carbon network in the samples with RGO contributing to good electronic conductivity, and the lack of a well-connected network in the samples without RGO, leading to poor conductivity as well as a large capacity decay.

\section{Acknowledgements}

We gratefully acknowledge funding from Department of Energy, Office of Basic Energy Sciences, EPSCoR Implementation award DE-SC0007074. We thank Chu Chen for help with the experiments. 


\section{References}

1. Liu, C.; Li, F.; Ma, L. P.; Cheng, H. M., Advanced Materials for Energy Storage. Advanced Materials 2010, 22 (8), E28-E62.

2. Tarascon, J.-M.; Armand, M., Issues and Challenges Facing Rechargeable Lithium Batteries. Nature 2001, 414 (6861), 359-367.

3. Etacheri, V.; Marom, R.; Elazari, R.; Salitra, G.; Aurbach, D., Challenges in the Development of Advanced Li-lon Batteries: A Review. Energy \& Environmental Science 2011, 4 (9), 3243-3262.

4. $\quad$ Chen, J., Recent Progress in Advanced Materials for Lithium lon Batteries. Materials 2013, 6 (1), 156-183.

5. Goodenough, J. B.; Park, K.-S., The Li-lon Rechargeable Battery: A Perspective. Journal of the American Chemical Society 2013, 135 (4), 1167-1176.

6. Scrosati, B.; Garche, J., Lithium Batteries: Status, Prospects and Future. Journal of Power Sources 2010, 195 (9), 2419-2430.

7. Noh, M.; Kwon, Y.; Lee, H.; Cho, J.; Kim, Y.; Kim, M. G., Amorphous CarbonCoated Tin Anode Material for Lithium Secondary Battery. Chemistry of materials 2005, $17(8), 1926-1929$.

8. Bu, P.; Liu, S.; Lu, Y.; Zhuang, S.; Wang, H.; Tu, F., Effects of Carbon Back on the Electrochemical Performance of Lithium-Organic Coordination Compound Batteries. Int J Electrochem Sci 2012, 7, 4617-4624.

9. Kim, J.; Kim, B.; Lee, J.-G.; Cho, J.; Park, B., Direct Carbon-Black Coating on Licoo 2 Cathode Using Surfactant for High-Density Li-Ion Cell. Journal of power sources 2005, 139 (1), 289-294.

10. Fransson, L.; Eriksson, T.; Edström, K.; Gustafsson, T.; Thomas, J. O., Influence of Carbon Black and Binder on Li-lon Batteries. Journal of power sources 2001, 101 (1), $1-9$.

11. Verdejo, R.; Bernal, M. M.; Romasanta, L. J.; Lopez-Manchado, M. A., Graphene Filled Polymer Nanocomposites. Journal of Materials Chemistry 2011, 21 (10), 3301-3310.

12. Chakraborty, I.; Bodurtha, K. J.; Heeder, N. J.; Godfrin, M. P.; Tripathi, A.; Hurt, R. H.; Shukla, A.; Bose, A., Massive Electrical Conductivity Enhancement of Multilayer Graphene/Polystyrene Composites Using a Nonconductive Filler. ACS applied materials \& interfaces 2014, 6 (19), 16472-16475.

13. Kim, H.; Abdala, A. A.; Macosko, C. W., Graphene/Polymer Nanocomposites. Macromolecules 2010, 43 (16), 6515-6530.

14. Larson, R. G., The Structure and Rheology of Complex Fluids. Oxford Univ Press: 1999; p 656 pp.

15. Wessel, R., Ball, R.C., Fractal Aggregates and Gels in Shear Flow. Physical Review A 1992, 46, R3008-R3011.

16. Zerda, T. W., Yang, H., Gerspacher, M., Fractal Dimension of Carbon-Black Particles. Rubber chemistry and technology 1992, 65, 130-136.

17. Li, J.; Ma, P.; Sze, C.; Kai, T.; Tang, B.; Kim, J. K. In Percolation Threshold of Polymer Nanocomposites Containing Graphite Nanoplatelets and Carbon Nanotubes, ICCM International Conferences on Composite Materials, 2007. 
18. Chen, Y.; Nie, M.; Lucht, B. L.; Saha, A.; Guduru, P. R.; Bose, A., High Capacity, Stable Silicon/Carbon Anodes for Lithium-Ion Batteries Prepared Using Emulsion-

Templated Directed Assembly. ACS applied materials \& interfaces 2014, 6 (7), 46784683.

19. Saha, A.; Nikova, A.; Venkataraman, P.; John, V. T.; Bose, A., Oil Emulsification Using Surface-Tunable Carbon Black Particles. ACS applied materials \& interfaces 2013, 5 (8), 3094-3100.

20. Magasinski, A.; Zdyrko, B.; Kovalenko, I.; Hertzberg, B.; Burtovyy, R.; Huebner, C. F.; Fuller, T. F.; Luzinov, I.; Yushin, G., Toward Efficient Binders for Li-lon Battery Si-Based Anodes: Polyacrylic Acid. ACS applied materials \& interfaces 2010, 2 (11), 3004-3010.

21. Chen, Y.; Xu, M.; Zhang, Y.; Pan, Y.; Lucht, B. L.; Bose, A., All-Aqueous Directed Assembly Strategy for Forming High-Capacity, Stable Silicon/Carbon Anodes for Lithium-Ion Batteries. ACS applied materials \& interfaces 2015, 7 (38), 2139121397.

22. Stone, H.; Brugh, M.; Hopkins, S.; Yoder, H.; Beard, C., Preparation of Inactivated Oil-Emulsion Vaccines with Avian Viral or Mycoplasma Antigens. Avian diseases 1978, 666-674.

23. Chung, G. C.; Jun, S. H.; Lee, K. Y.; Kim, M. H., Effect of Surface Structure on the Irreversible Capacity of Various Graphitic Carbon Electrodes. Journal of The Electrochemical Society 1999, 146 (5), 1664-1671.

24. Sharifi-Viand, A.; Mahjani, M.; Jafarian, M., Investigation of Anomalous Diffusion and Multifractal Dimensions in Polypyrrole Film. Journal of Electroanalytical Chemistry 2012, 671, 51-57. 